\title{
La realtà educativa e la ricerca-azione in pedagogia
}

\author{
Massimo Baldacci
}

Università degli Studi di Urbino, Dipartimento di Scienze dell'Uomo

doi: 10.7358/ecps-2014-009-bald

massimo.baldacci@uniurb.it

\section{EDUCATIONAL REALITY AND ACTION-RESEARCH IN EDUCATION}

\begin{abstract}
The article investigates the concept of «educational reality» with regard to an epistemology inspired by critical realism. The investigation firstly deals with the function of «educational reality" in action-research by gathering the its relations with "pedagogic problematicism" and with educational planning. In this context, action-research becomes an active cognitive strategy aimed at transforming educational reality. Secondly, the implications between educational reality and ontology are explored. The analysis leads to overcoming the unilateral formulations of exclusively natural and social ontologies in favour of a complex and critical ontological approach. Thirdly, the study analyses the consequences deriving from complex ontology on educational reality through action-research. Educational reality, being the result of previous educational processes, is objectified educational practice and, for the very fact that it is a product of preceding transformations, can undergo further changes. Therefore, action-research enables the implementation of a critical and scientific pedagogic realism. Since it is an active cognitive strategy, it enables an understanding of educational reality, hypothesizes the action of transformation of the educational situation and performs an objective control of the transformation obtained.
\end{abstract}

Keywords: Action-research, Complex ontology, Educational reality, Pedagogical realism, Problematicism. 


\section{PREMESSA}

In un precedente intervento su questa rivista, abbiamo cercato di mettere a punto un paradigma della ricerca pedagogica ispirato ad un atteggiamento "realista». Conformemente al razionalismo critico, al quale aderiamo, tale paradigma - pur ponendo al centro la categoria del realismo - integrava assunti teorici di differente natura: oggettivista, realista, costruttivista, pragmatista, ermeneutico, critico. In quella sede, la nostra preoccupazione era quella di presentare un'impostazione che pur senza cadere nelle forme dogmatiche di realismo, riuscisse ad evitare la deriva ermeneutica che affligge le odierne pratiche della ricerca-azione, rischiando di togliere loro qualsiasi affidabilità scientifica. Lo sforzo era perciò quello di definire un'epistemologia improntata a una sorta di realismo critico, innervato da una robusta vena oggettivista ma consapevole del senso e dei limiti della ricerca pedagogica e quindi scevro da dogmatismi.

Nel presente contributo - senza tornare a esporre il suddetto paradigma, al cui interno ci muoveremo - intendiamo mettere a fuoco il cruciale concetto di «realtà educativa». Questa espressione è fondamentale per due motivi: in primo luogo, la usiamo comunemente nel discorso pedagogico, pur lasciandola solitamente allo stato pre-analitico, ossia senza definirla, contentandoci della sua accezione intuitiva (che rinvia a una situazione di fatto non meglio specificata); in secondo luogo, un paradigma della ricerca pedagogica ispirato al realismo deve necessariamente presupporre l'esistenza di una «realtà educativa» rispetto alla quale si svolgono sia l'intervento educativo che l'indagine empirica.

Per indagare il concetto di "realtà educativa» toccheremo tre punti: (1) richiameremo sinteticamente le modalità di approccio al concetto di realtà educativa e la sua funzione entro la ricerca-azione; (2) cercheremo di cogliere alcune implicazioni ontologiche del concetto di realtà educativa; (3) infine, tenteremo di evidenziare le conseguenze di tali implicazioni sulla teoria della ricerca-azione.

Senza la pretesa di fornire una trattazione organica, stenderemo una serie di appunti di lavoro utili per una prima esplorazione della questione.

\section{IL CONCETTO DI REALTÀ EDUCATIVA E LA RICERCA-AZIONE}

Come abbiamo chiarito nel precedente contributo vi sono due modi di affrontare la questione della realtà educativa: uno pratico, l'altro teorico.

Sotto il profilo pratico, la realtà educativa si dà come qualcosa con cui si deve fare i conti, che pone difficoltà a cui occorre far fronte e che resiste alla 
realizzazione degli intenti educativi - e li può persino frustrare. Dall'angolazione teorica, la «realtà educativa» costituisce un concetto-base del discorso pedagogico. In questo senso, è significativa la posizione del problematicismo pedagogico ${ }^{1}$. Secondo questo indirizzo teorico, i canoni della scelta educativa sono costituiti dalla fedeltà alla ragione e dall'aderenza alla realtà. Il primo riguarda la razionalità rispetto alle finalità educative, il secondo concerne l'adeguatezza nei riguardi della concreta situazione educativa. In altre parole, occorre partire dalla realtà della situazione data, poiché la scelta educativa ha senso solo in riferimento a una specifico insieme di circostanze. La situazione educativa, cioè, possiede il carattere di una realtà indipendente dai nostri desideri, e con la quale deve fare i conti l'attuazione dei nostri progetti. Detto altrimenti, la situazione educativa è modificabile dall'intervento educativo, ma non è plasmabile a piacimento, è caratterizzata da proprie linee di for$\mathrm{za}$, da tendenze oggettive che occorre cogliere e comprendere per assicurare l'efficacia della scelta. Il canone dell'aderenza alla realtà nella scelta educativa distingue così una pedagogia scientifica e realistica da una pedagogia puramente utopica e velleitaria.

Ciò premesso, occorre collegare l'esigenza di una scelta educativa aderente alla realtà con la questione della ricerca educativa. Se ci si riferisce alla ricerca-azione il nesso è trasparente. Una scelta educativa organica si dà solo entro il quadro della progettazione educativa, nel cui ambito le decisioni tengono conto al tempo stesso degli scopi da raggiungere e delle concrete situazioni educative. Ma la ricerca-azione rappresenta una forma di ricercaprogetto $^{2}$. Difatti, se la progettazione educativa costituisce il modo di dare intenzionalità e razionalità alla pratica formativa, la ricerca-azione mira a dare "forma» di ricerca alla progettazione educativa, situandola entro lo schema problema-ipotesi di soluzione-messa alla prova-conferma/correzione. Ciò è possibile in quanto, al di là delle loro differenze di superficie, la ricerca-azione e la progettazione educativa condividono una medesima struttura profonda. In questo quadro, la definizione di tentativi di soluzione dei problemi educativi - proprio della ricerca-azione - viene a coincidere con la formulazione di un progetto educativo. Una scelta educativa aderente alla realtà si trova così trasportata dallo spazio della progettazione a quello della ricerca-azione educativa. Ma su questo trasferimento occorre dare qualche altra precisazione. Detto schematicamente, una ricerca-azione non prende le mosse soltanto da un problema che si dà come "tipico" pur presentandosi in una specifica e concreta situazione: essa muove anche da un sistema d'ipotesi, da un "modello", alla luce del quale viene concepito - in forma di progetto - il tentativo di

1 Bertin, 1975.

2 Becchi \& Vertecchi, 1986; Becchi, 1997. 
soluzione educativa. In assenza di un sistema d'ipotesi non si avrebbe ricerca ma solo azione, perché la ricerca empirica implica la messa alla prova d'ipotesi. Inoltre, un progetto in quanto tale non costituisce mai una soluzione trasferibile senza mediazioni a una situazione diversa da quella in cui è stato concepito. Difatti, proprio perché pensato in modo aderente a una specifica realtà educativa, esso risente anche delle contingenze di tale realtà e vari suoi aspetti potrebbero perciò non risultare aderenti a realtà educative differenti. Nella ricerca-azione, la trasferibilità riguarda sempre e solo il «modello educativo» (il sistema d'ipotesi) sperimentato. Il progetto entro il quale tale modello ha preso una forma specifica, aderente a una realtà particolare, costituisce piuttosto una sorta di "esemplare» attuativo del modello stesso. Pertanto, un medesimo modello si può incarnare in un'intera famiglia di esemplari progettuali che possiedono una comune struttura fondamentale pur differendo almeno in parte nelle azioni specifiche che prevedono in ragione delle differenti realtà educative entro le quali si devono calare. Tuttavia, la valutazione della ricerca-azione (del suo sistema d'ipotesi) si basa necessariamente sul genere di trasformazione della realtà educativa che si è prodotta in seguito all'attuazione del progetto. La ricerca-azione va concepita come una strategia di conoscenza attiva, che non si limita a interpretare la realtà educativa ma intende trasformarla. Nella ricerca-azione la conoscenza e la verità sono legate alla prassi, all'attività di trasformazione della situazione educativa. Una trasformazione reale, che come tale deve essere rilevabile oggettivamente (pur nei limiti che rendono ineliminabile una componente interpretativa di tale valutazione ${ }^{3}$ ) e non semplicemente intuita soggettivamente. E con questo si pone l'esigenza di passare al secondo punto: poiché nella ricerca-azione si richiede la conoscenza non di una realtà meramente data, ma di una trasformazione della realtà, occorre definire che genere di realtà sia la realtà educativa.

\section{LE IMPLICAZIONI ONTOLOGICHE DEL CONCETTO DI REALTÀ EDUCATIVA}

Domandarsi che genere di realtà sia la realtà educativa equivale a porsi un quesito "ontologico». Risponderemo in base a due coordinate: il canone dell'austerità ontologica formulato da Quine, e la distinzione tra ontologia natura-

3 Vd. Baldacci, 2013. Per i riferimenti epistemologici generali collegati: Popper, 1972; Preti, 1976; Bartley III, 1983; Lakatos, 1986; Rorty, 1998; Brandom, 2002. Per i quadri epistemologici della ricerca educativa: Domenici, 1993; Lucisano \& Salerni, 2004; Losito \& Pozzo, 2005; Giovannini \& Marcuccio, 2012. 
le e ontologia sociale. Il canone dell'austerità ontologica, di Quine ${ }^{4}$, costituisce una riedizione del celebre rasoio di Occam. In pratica, tale canone prescrive d'assumere solo gli impegni ontologici necessari per la significatività degli enunciati di un certo discorso. Se parliamo dell'attività educativa e della ricerca-azione come pratiche connesse alla trasformazione della realtà educativa, la sensatezza del discorso richiede di presupporre l'esistenza di qualcosa che chiamiamo "realtà educativa", ossia di una specifica regione della realtà a cui sia applicabile l'etichetta "educativa». Per altro, non è richiesto e non è opportuno postulare ulteriori caratteri di tale realtà se ciò non è strettamente necessario per la tenuta del discorso. Gli impegni ontologici sono inevitabili, ma vanno limitati: le ontologie "ricche» rischiano sempre di risultare arbitrarie in molte loro zone. E tuttavia, è bene ribadirlo, postulare l'esistenza di una realtà educativa "reale» è necessario per il discorso pedagogico, che altrimenti cadrebbe nell'assurdo.

Ciò premesso, per stabilire che genere di realtà sia la realtà educativa è opportuno rifarsi alla distinzione tra ontologia naturale e ontologia sociale 5 . Si tratta di decidere se la realtà educativa sia di pertinenza della prima o della seconda.

Se si assegna alla prima, all'ontologia naturale, essa cadrà sotto il principio della stabilità e della regolarità della natura, per cui la ricerca pedagogica tenderà a cogliere, di là dalle contingenze storico-sociali, le costanti naturali del processo educativo, basate sulla costituzione biologica dell'uomo. Così, si tenderà a ritenere che vi siano principi o leggi perenni dell'educazione che la pedagogia deve scoprire o ricostruire, e la ricerca assumerà un taglio nomotetico.

Se invece si vede la realtà educativa come una realtà di genere storicosociale, il quadro cambia completamente. In questo caso, infatti, è il divenire storico-sociale a creare le forme concrete e storico-relative dei processi educativi. Così, si tenderà a ritenere che i principi e le categorie dell'educazione siano astrazioni determinate e storicamente relative, e che esse vadano comprese entro il quadro storico-sociale.

Tale questione ontologica è complicata da due ulteriori elementi. Il primo è la possibilità di un'ontologia complessa ${ }^{6}$, sociale e naturale al tempo stesso, perché alcuni aspetti dell'educazione potrebbero essere inerenti alla sfera naturale, altri a quella sociale, e altri ancora essere difficilmente catalogabili. Le ontologie puramente naturali o sociali sarebbero allora unilaterali, e perciò destinate a generare impostazioni riduttive. La seconda è che spesso

4 Quine, 2005.

5 Ferraris, 2012.

6 Sulla «complessità» vd. Morin, 2005. 
gli impegni ontologici assunti in sede epistemologica rimangono impliciti, e agiscono così in modo incontrollato. Difatti, è impossibile sviluppare un discorso scientifico senza compiere assunzioni ontologiche. Ciò non significa che la ricerca pedagogica debba riferirsi a un'ontologia completa, tuttavia le è necessario almeno un orientamento ontologico generale ed esplicito, altrimenti il ricercatore rischia di essere influenzato in modo incontrollato da un presupposto ontologico implicito. Per il resto, una volta precisato questo orientamento generale, crediamo che ci si debba attenere al già citato principio di austerità ontologica.

In questa sede, assumeremo la posizione di un'ontologia complessa, sociale e naturale al tempo stesso, senza però fissare proporzioni tra tali aspetti. Un'ontologia complessa ha conseguenze rilevanti sulla teoria della ricerca, poiché l'epistemologia è almeno in parte vincolata dall'ontologia, in particolare rispetto all'adeguatezza del processo conoscitivo. Difatti, un'ontologia complessa rende guardinghi contro frettolose generalizzazioni nomotetiche, non solo rispetto alla necessità di un'evidenza empirica ampia e rappresentativa, ma al loro carattere nomotetico in quanto tale: alla pretesa di arrivare a leggi costanti e sovra-storiche, simili a leggi naturali, le quali corrono il rischio di reificare la struttura del processo educativo, riducendo a realtà meramente naturale una realtà storico-naturale, i cui principi potrebbero essere valevoli in modo puramente storico relativo ${ }^{7}$. Detto questo, non ci impegneremo però in ulteriori chiarimenti, aggiungendo soltanto che un'ontologia complessa esclude soluzioni semplicistiche del rapporto Natura/Storia. Ci dedicheremo invece a evidenziare alcune implicazione del lato storico-sociale di un'ontologia austera per la ricerca-azione educativa ${ }^{8}$.

\section{LE CONSEGUENZE DI UN'ONTOLOGIA COMPLESSA PER LA RICERCA-AZIONE}

Torniamo al concetto di realtà educativa e chiediamoci quali implicazioni abbia riferire questo concetto a un'ontologia complessa, che include in modo significativo un lato storico-sociale. Rimane immutata la capacità di resistere alle nostre intenzioni e alle nostre azioni da parte della realtà, il fatto che pur essendo almeno in parte modificabile, essa non si lascia plasmare a piacimen-

7 Per questi problemi, vd. Goldmann, 1981.

8 L'accettazione del principio di austerità ontologica, ossia di un'ontologia minimale, non impedisce di ritenere che un certo livello del discorso pedagogico sia del tutto libero da ipoteche ontologiche. Nel razionalismo critico, questo livello è quello delle idee trascendentali, il cui valore non è costitutivo ma puramente regolativo. Vd. Banfi, 1926. 
to. Tuttavia, mentre la realtà naturale è indipendente dall'uomo (nel senso che sussiste di per sé, anche se l'uomo la modifica in funzione dei propri bisogni), la realtà storico-sociale di cui è intessuta l'educazione è solo relativamente indipendente dall'attività dell'uomo.

Cerchiamo di chiarire. Se nel campo in questione l'attività umana consiste nella prassi educativa, la realtà educativa è costituita da prassi educativa oggettivata, ossia rappresenta il prodotto cristallizzato e sedimentato di processi educativi precedenti ${ }^{9}$. Tali prodotti consistono prima di tutto in stati mentali consolidati dei soggetti implicati nella situazione educativa, quali: conoscenze, abilità, abiti mentali; ma anche nelle caratteristiche socio-culturali dell'ambiente educativo complessivo. In quanto prassi oggettivata, la realtà educativa è solo relativamente indipendente dall'uomo, può resistere all'azione educativa contingente, ma si presenta costitutivamente modificabile. La realtà educativa esistente, cioè, è null'altro che il prodotto della trasformazione di una realtà educativa preesistente, ed è oggetto di un ulteriore potenziale cambiamento. In altri termini, è la prassi educativa che fa la realtà educativa, nel senso che tende a farsi tale oggettivandosi. Ovviamente, che la realtà educativa sia il prodotto di una trasformazione operata dalla prassi, non implica il totale cambiamento delle cose. Mentre trasforma alcuni aspetti della realtà educativa, la prassi ne consolida altri, e sono questi aspetti cristallizzati e sedimentati nel tempo a offrire una maggiore resistenza al cambiamento, fino al punto di poter determinare il fallimento di un progetto educativo.

Stando così le cose, nella ricerca-azione, la conoscenza e la verità sono legate alla prassi, all'azione di trasformazione della situazione educativa. Difatti, la ricerca-azione è una strategia cognitiva attiva, perciò entro la sua cornice la conoscenza non è definibile come un mero rispecchiamento della realtà educativa, come pura fedeltà della descrizione allo stato di cose, ma al contrario prende corpo nell'attività volta a conformare lo stato di cose esistente al disegno educativo. Pertanto, la verità dell'ipotesi progettuale consiste nel suo verificarsi, nella sua efficacia rispetto alla trasformazione progettata della realtà educativa. In altri termini, nella ricerca-azione l'accordo tra ipotesi e realtà è una questione pratica, che può essere controllato solo nell'ambito della prassi stessa: il vero è il verificato ${ }^{10}$. Beninteso, controllare ciò che si è verificato non è però materia di opinione, ma di conoscenza oggettiva della realtà prodotta, della trasformazione ottenuta. E questo istituisce la forma di correlazione soggetto/oggetto propria di un'epistemologia critico-realista della ricerca-azione. La realtà educativa iniziale costituisce una realtà oggettiva, pur essendo prassi educativa cristallizzata si dà come indipendente dai voleri

9 Marx, 1975; Fichte, 2008.

10 Vd. Dewey, 1998. 
dell'educatore, ed è suscettibile di resistere ai suoi disegni (e qui abbiamo il primo aspetto del realismo pedagogico: il realismo rispetto alla situazione educativa). Il progetto educativo è un atto creativo della soggettività dell'educatore, ma per non essere arbitrario o velleitario non deve semplicemente essere fedele a certe finalità educative, bensì deve porsi come aderente alla realtà educativa esistente pur proponendosi di trasformarla (e qui abbiamo il secondo aspetto del realismo pedagogico: il realismo del progetto rispetto alla realtà esistente). Infine, la trasformazione della realtà educativa secondo il disegno pedagogico, non è una questione soggettiva e nemmeno puramente intersoggettiva (non basta il giudizio della maggioranza per validare l'esito di un progetto), bensì è materia di conoscenza oggettiva, sebbene non priva di aspetti ermeneutici (e qui abbiamo il terzo aspetto del realismo pedagogico: l'oggettività della valutazione degli esiti del progetto).

Certamente questi tre aspetti del realismo pedagogico hanno un significato diverso, ma che rinvia alla rilevanza della categoria della «realtà». Nel primo caso, riferito alla situazione educativa esistente, il realismo ha il significato di indipendenza di tale situazione dalla soggettività dell'educatore. Nel secondo caso, il realismo costituisce un attributo della creazione progettuale soggettiva dell'educatore, circoscrivendo la sua soggettività all'ambito ontologico (il progetto è costruito dall'educatore), ossia escludendone quello epistemologico: la progettazione è fatta dal soggetto, ma non deve essere soggettiva, bensì serbare il senso della realtà rispetto alla concretezza della situazione esistente (ossia, mantenersi realistica). Infine, nel terzo caso il realismo costituisce una caratteristica del processo conoscitivo della trasformazione della situazione, che deve attestare oggettivamente se la trasformazione prodotta è coerente col disegno pedagogico (ossia, se la situazione è realmente cambiata nel modo voluto).

Per concludere, un realismo critico basato sul nesso tra prassi e realtà educativa si pone in alternativa tanto al fatalismo quanto all'utopismo ${ }^{11}$.

Per il fatalismo la realtà educativa è qualcosa di dato, che è molto difficile modificare. Di solito questo atteggiamento è legato a forme di pseudonaturalismo pedagogico, che considera "naturale» che le cose stiano in una certa maniera o vadano in un certo modo. Un realismo critico, poiché considera la realtà come prassi oggettivata la ritiene costitutivamente modificabile, sebbene a certe condizioni, con certi modi e con certi tempi. Pertanto, esso porta a scongiurare il fatalismo, a favore di un atteggiamento attivo e fattivo.

Secondo l'utopismo, invece, la realtà educativa è plasmabile a piacimento, basta solo concepire la forma da darle e agire attivamente per concretarla. Spesso questo atteggiamento è connesso a forme di romanticismo pedagogico, inclini al volontarismo e talvolta al titanismo (l'educatore concepito come

11 Putnam, 1995; Nagel, 1999. 
demiurgo, investito della missione di redimere la società). Un realismo critico, poiché considera la prassi educativa oggettivata come una realtà indipendente dai voleri dell'educatore, non la ritiene modificabile a piacimento ma solo a certe condizioni, da comprendere nella loro specificità situata, e la cui inosservanza porta al fallimento del disegno pedagogico. Pertanto, esso conduce ad evitare l'utopismo velleitario, a favore di un atteggiamento realistico.

Contro il fatalismo e l'utopismo, si può pertanto definire l'impianto generale di un realismo pedagogico di marca critica e scientifica: comprendere la realtà educativa esistente nella sua oggettività, per concepirne la trasformazione possibile e le sue condizioni reali; intervenire attivamente su tali condizioni per cambiare la situazione educativa nel modo voluto, controllando che si modifichi realmente in tale modo ${ }^{12}$.

\section{RIFERIMENTI BIBLIOGRAFICI}

Baldacci, M. (2012). Trattato di pedagogia generale. Roma: Carocci.

Baldacci, M., \& Frabboni, F. (a cura di). (2013). Manuale della ricerca educativa. Torino: UTET.

Banfi, A. (1926). Principi di una teoria della ragione. Torino: Paravia (trad. it., Roma: Editori Riuniti, 1967).

Bartley III, W. W. (1983). Come demarcare la scienza dalla metafisica. Roma: Borla.

Becchi, E. (1997). Sperimentare nella scuola. Firenze: La Nuova Italia.

Becchi, E., \& Vertecchi, B. (a cura di). (1986). Manuale critico della sperimentazione e della ricerca educativa. Milano: Franco Angeli.

Bertin, G. M. (1975). Educazione alla ragione. Roma: Armando.

Brandom, R. B. (2002). Articolare le ragioni. Milano: Il Saggiatore.

Dewey, J. (1948). Liberalism and social action. Boston: Beacon (trad. it., Rifare la filosofia. Roma: Donzelli, 1998).

Domenici, G. (1993). Manuale della valutazione scolastica. Roma - Bari: Laterza.

Ferraris, M. (2012). Manifesto del nuovo realismo. Bari - Roma: Laterza.

Fichte, J. G. (2008). Scritti sulla dottrina della scienza (1794-1804). Milano: Mondadori.

Giovannini, M. L., \& Marcuccio, M. (2012). Ricerca sulla valutazione di un progetto scolastico innovativo. Milano: Franco Angeli.

Goldmann, L. (1981). Scienze umane e filosofia. Milano: Feltrinelli.

12 Baldacci, 2012. 
Lakatos, I. (1986). La falsificazione e la metodologia dei programmi di ricerca scientifici. In I. Lakatos \& A. Musgrave (a cura di), Critica e crescita della scienza. Milano: Feltrinelli.

Losito, B., \& Pozzo, G. (2005). La ricerca azione. Roma: Carocci.

Lucisano, P., \& Salerni, A. (2004). Metodologia della ricerca in educazione e formazione. Roma: Carocci.

Marx, K. (1845). Tesi su Feuerbach. In U. Cerroni (a cura di), Il pensiero di Marx (pp. 142-146). Roma: Editori Riuniti.

Morin, E. (2005). Introduction à la pensée complexe. Paris: Seuil.

Nagel, T. (1999). Lultima parola. Contro il relativismo. Milano: Feltrinelli.

Popper, K. (1972). Congetture e confutazioni. Bologna: Il Mulino.

Preti, G. (1976). Saggi filosofici, Vol. 1. Firenze: La Nuova Italia.

Putnam, H. (1995). Realismo dal volto umano. Bologna: Il Mulino.

Quine, W. V. O. (2005). Dal punto di vista logico. Milano: Cortina.

Rorty, R. (1998). La filosofia e lo specchio della natura. Milano: Bompiani.

\section{RiassunTO}

L'articolo indaga il concetto di "realtà educativa» inteso nell'ambito di un'epistemologia improntata al realismo critico. Lindagine affronta, in primo luogo, la funzione della "realtà educativa" nella ricerca azione, cogliendone le relazioni con il "problematicismo pedagogico" e con la progettazione educativa. La ricerca azione, in questo quadro, diventa una strategia cognitiva attiva tesa a trasformare la realtà educativa. In secondo luogo vengono esplorate le implicazioni fra realtà educativa e ontologie. L'analisi conduce al superamento delle impostazioni unilaterali delle ontologie esclusivamente naturali e sociali a favore di un approccio ontologico complesso e critico. In terzo luogo, vengono prese in esame le conseguenze portate dall'ontologia complessa sulla realtà educativa per mezzo della ricerca azione. La realtà educativa, in quanto esito dei processi educativi precedenti, è prassi educativa oggettivata e, proprio in quanto prodotto delle trasformazioni precedenti, può diventare oggetto di ulteriori cambiamenti. La ricerca azione, quindi, consente di attuare il realismo pedagogico critico e scientifico. In quanto strategia cognitiva attiva permette la comprensione della realtà educativa, ipotizza l'azione di trasformazione della situazione educativa ed effettua il controllo oggettivo della trasformazione ottenuta.

Parole chiave: Ontologia complessa, Problematicismo, Realismo pedagogico, Realtà educativa, Ricerca azione. 